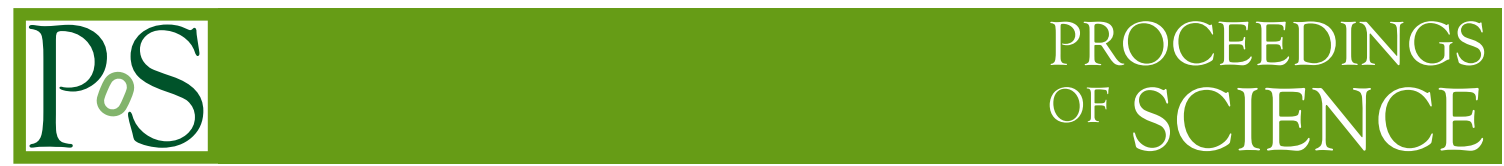

\title{
Diffusive scaling and the high energy limit of DDIS
}

\author{
Yoshitaka Hatta* \\ RIKEN BNL Research Center, Brookhaven National Laboratory, Upton, NY 11973, USA \\ E-mail: hatta@quark.phy.bnl.gov
}

\begin{abstract}
After reviewing the recent developments on the high energy evolution equation beyond the BKJIMWLK equation, we discuss their impact on the inclusive diffractive cross section in DIS. Due to the Pomeron loop effects, the cross section exhibits a new scaling law-the 'diffusive' scaling which eventually replaces the geometric scaling as predicted by the BK-JIMWLK equation.
\end{abstract}

DIFFRACTION 2006 - International Workshop on Diffraction in High-Energy Physics September 5-10 2006

Adamantas, Milos island, Greece

* Speaker. 


\section{Nonlinear evolution equations}

One of the best hopes for unitalizing the BFKL Pomeron within perturbative QCD is to derive nonlinear evolution equations by summing gluon recombination diagrams. The most complete equation of this kind to date is the Balitsky-JIMWLK equation[1]:

$$
\frac{\partial\left\langle T_{x y}\right\rangle}{\partial Y}=\frac{\bar{\alpha}_{s}}{2 \pi} \int d z \frac{(\boldsymbol{x}-\boldsymbol{y})^{2}}{(\boldsymbol{x}-\boldsymbol{z})^{2}(\boldsymbol{z}-\boldsymbol{y})^{2}}\left(\left\langle T_{x z}\right\rangle+\left\langle T_{z y}\right\rangle-\left\langle T_{x y}\right\rangle-\left\langle T_{x z} T_{z y}\right\rangle\right),
$$

where $Y$ is the rapidity and $T_{x y}$ is the scattering amplitude of a color dipole of size $r=|\boldsymbol{x}-\boldsymbol{y}|$ off a dense target evaluated in the eikonal approximation

$$
T_{x y}=1-\frac{1}{N_{c}} \operatorname{Tr}\left\{\mathrm{V}_{\boldsymbol{x}} \mathrm{V}_{\boldsymbol{y}}^{\dagger}\right\}
$$

with

$$
V_{\boldsymbol{x}}=\operatorname{Pexp}\left(i g \int d x^{-} A^{+}\left(x^{-}\right)\right)
$$

$A^{+}$describes the target color field which is typically $\sim \mathscr{O}(1 / g)$ in the saturation regime and $\langle\ldots\rangle$ in Eq. (1.1) denotes an averaging over the target field in the framework of the color glass condensate (CGC) formalism. In fact, Eq. (1.1) is the first equation of an infinite hierarchy which schematically reads

$$
\begin{aligned}
& \frac{\partial\left\langle T^{(2)}\right\rangle}{\partial Y}=\bar{\alpha}_{s}\left(\left\langle T^{(2)}\right\rangle-\left\langle T^{(3)}\right\rangle\right), \\
& \frac{\partial\left\langle T^{(3)}\right\rangle}{\partial Y}=\bar{\alpha}_{s}\left(\left\langle T^{(3)}\right\rangle-\left\langle T^{(4)}\right\rangle\right), \\
& \ldots
\end{aligned}
$$

where $T^{(n)}$ is the scattering amplitude for $n$ dipoles and we suppressed convolution with the BFKL kernel and numerical factors. With an additional assumption of factorization, $\langle T T\rangle=\langle T\rangle\langle T\rangle$, Eq. (1.1) becomes a closed equation known as the Balitsky-Kovchegov (BK) equation. The BKJIMWLK equation has been intensively studied both analytically and numerically. At fixed impact parameter $\boldsymbol{b}=\frac{\boldsymbol{x}+\boldsymbol{y}}{2}$, the solution looks like Fig. 1 as a function of $\ln 1 / r^{2}$.

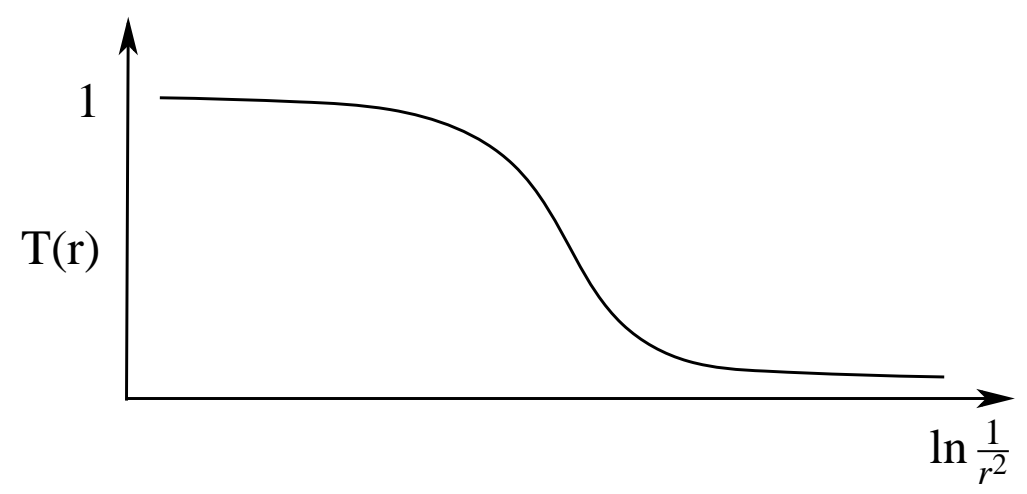

Figure 1: Schematic solution of the BK-JIMWLK equation plotted as a function of $\ln 1 / r^{2}$. 


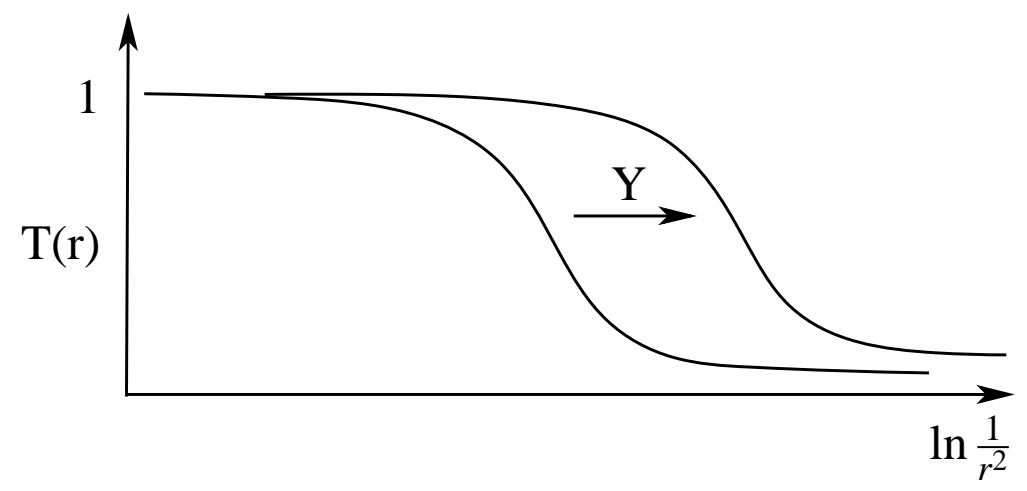

Figure 2: Schematic solution of the BK-JIMWLK equation plotted as a function of $\ln 1 / r^{2}$.

When $r \rightarrow 0, T(r)$ goes to zero due to color transparency, while at large $r$, it saturates to the black disc limit value $T(r) \rightarrow 1$ thanks to the nonlinear term $\sim T^{2}$ in the evolution equation. At intermediate values of $r$, a wavefront is formed. The location of this front (the onset of strong scattering) defines the saturation momentum $Q_{s}(Y) \propto e^{\lambda Y}: T\left(r=1 / Q_{s}(Y)\right)=$ const. $\sim \mathscr{O}(1)$. A very important property of this solution is that it shows geometric scaling. Namely, the dipole scattering amplitude, which is a priori a function of two variables $r$ and $Y$, depends only on a single variable $r Q_{s} ; T(r, Y) \approx\left(r^{2} Q_{s}^{2}(Y)\right)^{\gamma},(\gamma \approx 0.63)$ in a wide region above the saturation momentum $r \geq 1 / Q_{s}(Y)$. This means that, as the rapidity increases, the front is parallel transported to the right without changing its shape. There is a nice way of understanding this 'travelling wave' behavior of the solution. After an approximation to the BFKL kernel and some changes of variables, the BK equation becomes identical to the Fisher-Kolmogorov-Petrovsky-Piscounov (FKPP) equation which is well known in statistical physics [2]. There it has been known for some time that the solution to this equation is of a travelling wave type. This observation turns out to be crucial for going beyond the BK-JIMWLK equation as discussed in section 3.

\section{Prediction for (D)DIS: 'Mean field' results}

The dipole scattering amplitude $T(r)$ directly enters observed cross sections at small- $x$ via the dipole factorization formula $\boldsymbol{b}$,

$$
\frac{d \sigma_{T / L}}{d \boldsymbol{b}}\left(Y, Q^{2}\right)=2 \int d z d r\left|\Psi_{T / L}\left(z, r, Q^{2}\right)\right|^{2}\langle T(r)\rangle_{Y},
$$

where $\Psi$ is the QED amplitude $\gamma_{T / L}^{*} \rightarrow q \bar{q}$ and $z$ is the longitudinal momentum fraction taken by the quark. Plugging the solution $\langle T(r)\rangle$ of the BK-JIMWLK equation into Eq. (2.1), one sees that, when $Q>Q_{s}$, the $r$ integral is dominated by $1 / Q<r<1 / Q_{s}$. The geometric scaling of $\langle T(r)\rangle$ is taken over by the geometric scaling of $\sigma, \sigma\left(Y, Q^{2}\right)=\sigma\left(Q^{2} / Q_{s}^{2}(Y)\right)$. Similarly, for the inclusive diffraction one gets, at small $\beta$,

$$
\frac{d \sigma_{T / L}^{D}}{d \boldsymbol{b}}\left(Y, Y_{0}, Q^{2}\right)=\int d z d r\left|\Psi_{T / L}\left(z, r, Q^{2}\right)\right|^{2} P^{D}\left(Y, Y_{0}, Q^{2}\right),
$$

where $Y-Y_{0}=\ln 1 / \beta$. Our definition is such that $Y_{0}$ is the minimal rapidity gap. So the diffractive probability $P^{D}$ is integrated over events whose rapidity gaps are equal to, or larger than $Y_{0}$. The 
usual diffractive structure function is obtained by taking a $\beta$-derivative, $F^{D(3)} \propto d / d(\ln 1 / \beta) \sigma^{D}$. $P^{D}$ naturally splits into the elastic diffraction $P_{e l}^{D}$ and the inelastic diffraction (diffractive dissociation) $P_{\text {inel }}^{D}$ which are given, respectively, by

$$
\begin{aligned}
P_{e l}^{D} & =\langle T(r)\rangle^{2}+\ln \frac{1}{\beta} \frac{\bar{\alpha}_{s}}{\pi} \operatorname{Re} \int d z M_{x y z}\left\langle T_{x y}\right\rangle\left\langle T_{x z}+T_{z y}-T_{x y}-T_{x z} T_{z y}\right\rangle, \\
P_{\text {inel }}^{D} & =\ln \frac{1}{\beta} \frac{\bar{\alpha}_{s}}{2 \pi} \int d z M_{x y z}\left|\left\langle T_{x z}+T_{z y}-T_{x y}-T_{x z} T_{z y}\right\rangle\right|^{2},
\end{aligned}
$$

where we have limited ourselves to the $q \bar{q}$ and $q \bar{q} g$ components of the virtual photon and used the large $N_{c}$ approximation to represent the $q \bar{q} g$ state as a two dipole system. Note that 'elastic' or 'inelastic' refers to the relative normalization of various Fock components of the virtual photon before and after the scattering (the Good-Walker picture). Therefore, the $q \bar{q} g$ state also contributes to the elastic part. When one tries to evaluate the rhs. of Eq. (2.3) by substituting $\langle T(r)\rangle \sim\left(r^{2} Q_{s}^{2}(Y)\right)^{\gamma}$, one immediately notices that small size dipoles are suppressed due to the factor $\langle T(r)\rangle^{2} \sim\left(r^{2}\right)^{2 \gamma}$. Correspondingly, the $r$ integral is dominated by large dipoles of size $r \sim 1 / Q_{s}(Y)>1 / Q$ which is still perturbative if $Y$ is large. (Note that without saturation, inclusive diffraction is a soft process dominated by large dipoles of size $r \sim 1 / \Lambda_{Q C D}$.) Other terms of Eq. (2.3) are similarly computed and the result is that $P_{\text {inel }}^{D}$ dominates over $P_{e l}^{D}$ at small $\beta$ for $Q>Q_{s}$. On the other hand, for $Q<Q_{s}$, $P_{\text {inel }}^{D}$ goes to zero since $T \rightarrow 1$.

\section{Beyond the BK-JIMWLK: Pomeron loop effects}

Recently there has been growing evidence that the gluon number fluctuation developed in the dilute regime plays an important role for the asymptotic behavior of the scattering amplitude. Such effects are not included in the BK-JIMWLK equation which contains only the gluon recombination diagrams, Fig. 3(a), but are due to the gluon splitting diagrams Fig. 3(b). A priori, these diagrams are important when the target is 'dilute'. However, even in DIS where the target eventually becomes saturated, small dipoles $r \rightarrow 0$ undergo weak scattering $T(r) \sim \alpha_{s}^{2}$, and in this region one cannot neglect the gluon splitting process. It turns out that the dynamics of this tail part dominates the asymptotic propagation of wavefronts. Iteration of vertices in Figs. 3(a) and 3(b) (and more generally, 3(c)) in the $t$-channel gives rise to loops of reggeons, or Pomerons. At the moment complete treatment of Pomeron loop effects in the evolution equation is not known. However, in the DIS case there have been already some significant progresses which we are going to review.

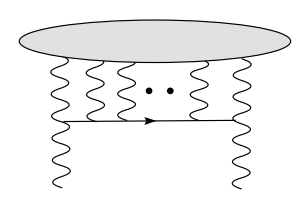

(a)

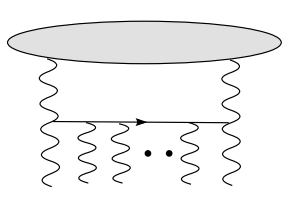

(b)

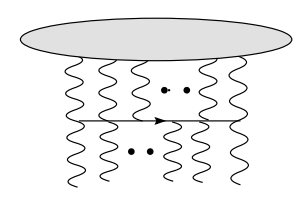

(c)

Figure 3: Quantum evolution of the target (represented by a blob): (a) Gluon recombination (b) Gluon splitting (c) Both the gluon recombination and the splitting

The connection between Fig. 3(b) and the gluon number fluctuation is best illustrated by the Monte-Carlo simulation [3] of the evolution of an onium in the dipole model at large $N_{c}$. This 
process is, in a sense, an extreme opposite of saturation: It contains only Fig. 3(b) in the form of dipole splitting, but not Fig. 3(a). As the rapidity is increased, each dipole in an onium splits into two new dipoles with certain probability computed in perturbation theory. It was observed in [3] that, after some evolution in rapidity, the number of dipoles of a fixed size in the onium wavefunction greatly fluctuates event-by-event. As the rapidity is further increased, the saturation effects set in and the dipole model breaks down. But one can easily imagine that the gluon number fluctuations developed in the early stages of the evolution persists in the saturation environment in the form of saturation momentum fluctuation, since the saturation momentum is a measure of gluon number density. This expectation is indeed the case. In [4], it was shown that, within the large $N_{c}$ approximation, the effect of Fig. 3(b) can be simulated by adding Gaussian noise to the rhs. of the BK-JIMWLK equation. In momentum space,

$$
\frac{\partial T(\boldsymbol{k})}{\partial Y}=\bar{\alpha}_{s} H_{B K-J I M W L K} \otimes T(\boldsymbol{k})+\bar{\alpha}_{s} \sqrt{\alpha_{s}^{2} T(\boldsymbol{k})} v(\boldsymbol{k})
$$

where $v$ is random noise with $\left\langle v(\boldsymbol{k}) v\left(\boldsymbol{k}^{\prime}\right)\right\rangle \sim 1 / \bar{\alpha}_{s} \boldsymbol{\delta}^{(2)}\left(\boldsymbol{k}-\boldsymbol{k}^{\prime}\right)$. One sees that the newly added term is important when $T \sim \alpha_{s}^{2}$. Eq. (3.1) is known in statistical physics as the stochastic-FKPP equation, and general features of its solution is known. In particular, numerical simulations show that the front position becomes a random variable after some evolution even if one starts with a single initial condition. In QCD, this means that the saturation momentum $Q_{s}$ becomes a random variable event-by-event, which is in qualitative agreement with what one would expect after summing the gluon splitting diagrams Fig. 3(b) as explained above. Moreover, the probability distribution of $Q_{s}$ is Gaussian in $\ln Q_{s}^{2}$, with the variance growing like $\sqrt{Y}$.

$$
P\left(Q_{s}\right) \sim \exp \left(-\frac{\ln ^{2}\left(Q_{s}^{2} /\left\langle Q_{s}\right\rangle^{2}\right)}{D Y}\right)
$$

where $\left\langle Q_{s}\right\rangle(Y)$ is the average saturation momentum. The observed amplitude is given by an average over the ensemble of $Q_{s}$

$$
\langle T(r)\rangle=\int d Q_{s} P\left(Q_{s}\right) T\left(r Q_{s}\right) \approx \frac{1}{2} \operatorname{Erf}\left(\frac{\ln 1 /\left(r^{2} \bar{Q}_{s}^{2}\right)}{\sqrt{D Y}}\right), \quad\left(\sqrt{D Y} \ll \ln 1 /\left(r^{2} \bar{Q}_{s}^{2}\right) \ll D Y\right)
$$

where $\operatorname{Erf}(x)$ is the error function. An important point here is that, though each event in the ensemble has the geometric scaling property $T\left(r Q_{s}\right)$, this property is lost in the averaged quantity because of the additional $Y$ dependence coming from the dispersion of the front. Instead, Eq. (3.3) exhibits a new type of scaling, the diffusive scaling-the scattering amplitude depends on $Y$ and $r$ via the combination $\ln \left(r^{2} \bar{Q}_{s}^{2}(Y)\right) / \sqrt{Y}$.

\section{Impact on DDIS}

Now that we have the novel behavior of the scattering amplitude, Eq. (3.3), predictions for DDIS should be updated and compared with the 'mean field' behavior of section 2 . The details of the calculation are presented in [5]. The immediate consequence of Eq. (3.3) is that small dipoles are no longer suppressed. Indeed, within the diffusive scaling window $\sqrt{D Y} \ll \ln 1 /\left(r^{2} \bar{Q}_{s}^{2}\right) \ll D Y$, the error function falls like Gaussian 


$$
\langle T(r)\rangle \sim \exp \left(-\ln ^{2}\left(r^{2} \bar{Q}_{s}^{2}\right) / D Y\right)
$$

and squaring it is not a big effect unlike the case of the mean field result $T(r) \sim\left(r^{2} Q_{s}^{2}\right)^{\gamma}$. Therefore, the $r$ integral in Eq. (2.2) is dominated by $r \sim 1 / Q \ll 1 / \bar{Q}_{s}$-diffraction becomes a hard process!

It is straightforward to evaluate other terms in Eq. (2.3). After the convolution with the photon wavefunction, the result again shows the diffusive scaling with the variable $Z \equiv \ln \left(Q^{2} / \bar{Q}_{s}^{2}\right) / \sqrt{D Y}$

$$
\frac{d \sigma^{D}}{d \boldsymbol{b}} \approx \frac{d \sigma_{e l}^{D}}{d \boldsymbol{b}} \propto \sqrt{D Y} \frac{e^{-2 Z^{2}}}{Z^{3}}, \quad\left(\sqrt{D Y} \ll \ln 1 /\left(Q^{2} / \bar{Q}_{s}^{2}\right) \ll D Y\right)
$$

Note that $P_{e l}^{D}$ dominates over $P_{\text {inel }}^{D}$ even when $Q^{2} \gg\left\langle Q_{s}\right\rangle^{2}{ }^{1}$ As already remarked in section 2, this phenomenon is expected in the saturation region $Q^{2} \ll\left\langle Q_{s}\right\rangle^{2}$. The physical reason why this happens also for $Q^{2} \gg\left\langle Q_{s}\right\rangle^{2}$ is the fluctuation of $Q_{s}$ - There are events in the ensemble whose saturation momentum is $Q_{s} \sim Q \sim 1 / r \gg\left\langle Q_{s}\right\rangle$. These events are rare, coming from the upper tail of the gaussian distribution Eq. (3.2). But since $Q_{s} \sim 1 / r$, the scattering is strong $T \sim \mathscr{O}(1)$. On the other hand, typical events with $Q_{s} \sim\left\langle Q_{s}\right\rangle$ are more frequent, but the cross section is small $T \ll 1$ for these events. It turns out that the former wins after averaging over $Q_{s}-$ cross section is dominated by rare fluctuations ('hot spots') in the ensemble which has unusually large saturation momentum $Q_{s} \sim Q \gg\left\langle Q_{s}\right\rangle$. In this way the fluctuation effects 'push up' the saturation physics well above the average saturation momentum $\left\langle Q_{s}\right\rangle$. The dominance of $P_{e l}^{D}$ over $P_{\text {inel }}^{D}$ is an example of this general tendency.

\section{Acknowledgements}

I would like to thank the organizers of Diffraction 2006 for invitation. This work has been done in collaboration with E. Iancu, C. Marquet, G. Soyez and D. Triantafyllopoulos.

\section{References}

[1] I. Balitsky, Nucl. Phys. B463 (1996) 99; J. Jalilian-Marian, A. Kovner, A. Leonidov and H. Weigert, Phys. Rev. D59 (1999) 014014; E. Iancu, A. Leonidov and L. McLerran, Nucl. Phys. A692 (2001) 583; Y. V. Kovchegov Phys. Rev. D 60 (1999) 034008.

[2] S. Munier and R. Peschanski, Phys. Rev. Lett. 91 (2003) 232001.

[3] G. P. Salam, Nucl. Phys. B 449 (1995) 589.

[4] E. Iancu and D. N. Triantafyllopoulos, Nucl. Phys. A 756 (2005) 419.

[5] Y. Hatta, E. Iancu, C. Marquet, G. Soyez and D. N. Triantafyllopoulos, Nucl. Phys. A 773 (2006) 95.

\footnotetext{
${ }^{1}$ Remember that $P^{D}\left(Y, Y_{0}\right)$ is integrated over $\beta$ from $\beta=1$ to $\beta=e^{-\left(Y-Y_{0}\right)} . P_{e l}^{D}$ receives contribution from $\beta \sim 1$ while $P_{\text {inel }}^{D}$ from $\beta \ll 1$. The derivative $d P^{D} / d \beta$ at small $\beta$ is always dominated by inelastic diffraction.
} 